\title{
Macroscopic quantum effects in a strongly driven nanomechanical resonator
}

\author{
V. Peano and M. Thorwart \\ Institut für Theoretische Physik IV, Heinrich-Heine-Universität Düsseldorf, \\ Universitätsstraße 1, D-40225 Düsseldorf, Germany
}

(Dated: September 16, 2018)

\begin{abstract}
We investigate the nonlinear response of a vibrating suspended nanomechanical beam on external periodic driving. The amplitude of the fundamental transverse mode behaves thereby like a weakly damped quantum particle in a driven anharmonic potential. Upon using a Born-Markovian master equation, we calculate the fundamental mode amplitude for varying driving frequencies. In the nonlinear regime, we observe resonances which are absent in the corresponding classical model. They are shown to be associated with resonant multi-phonon excitations. Furthermore, we identify resonant tunneling in a dynamically induced bistable effective potential.

PACS numbers: 03.65.-w, 62.25.+g, 62.30.+d, 03.65.Xp
\end{abstract}

\section{INTRODUCTION}

The ongoing progress in miniaturization of microscale devices allows nowadays to fabricate mechanical resonators on the nanometer scale $\stackrel{1}{\underline{1}}$. To realize them in form of transversely vibrating beams, lithographically patterned doubly clamped suspended beams 2.3.4.5.6.7.8.9.10.11 are designed, but also suspended carbon nanotubes display mechanical vibrations ${ }^{12}$ (see also Ref. 13 ). Beyond applications as electrometers 2.3 , for detecting ultrasmall forces and displacements 5.11 , or radio-frequency signal processing ${ }^{7}$, the nanomechanical devices also allow to investigate fundamental physical phenomena. In particular, due to their small size, the crossover from the classical to the quantum regime is of interest 14.15 . The quantum behavior will be due to a macroscopic number of particles whose coherence is disturbed by the interaction with the environment. Promising progress in approaching the quantum regime experimentally has been reported recently 8.9.11.

In this work, we propose a straight-forward way to reveal the quantum behavior of nanoresonators via the nonlinear response of a nanobeam to an external $a c$-driving. Mechanical excitation frequencies of nanodevices have been measured via $a c$-driving 3 ,4.5.6.7.8.9,10,11.16 . We consider the amplitude of the fundamental transverse vibrating mode of a doubly clamped suspended nanobeam under longitudinal compression. The compression allows to control the degree of nonlinearity of the nanoresonator. Starting from a continuum mode1 ${ }^{14.15}$ (see Ref. 17 for the classical counterpart), the interacting field theory closed to the Euler buckling instability can be reduced to the dynamics of a quantum particle in an anharmonic potential. By exciting the beam to transverse vibrations, its non-linear response can be determined. The environment is included by coupling the system to a harmonic Ohmic bath ${ }^{18}$. For weak driving, the response will be maximal at the eigenfrequency of the nanobeam and similar to that of the analogous classical system. However, as we show below, for strong driving, the non-linear response of the quantum system is qualitatively different from its classical counterpart which is the standard Duff- ing system 17 . This feature allows to separate both the classical and the quantum regime. In particular, we find distinct resonances in the dependence of the amplitude of the fundamental mode on the driving frequency. They can be interpreted as resonant multi-phonon excitations and are determined by avoided level crossings in the Floquet spectrum. In turn, we identify a separation of time scales and resonant tunneling in the dynamically induced bistable effective potential.

\section{EFFECTIVE SINGLE-PARTICLE MODEL STARTING FROM A CONTINUOUS MODEL}

We start from the normal mode description of an elastic rectangular beam of length $l$, width $w$ and thickness $d$ such that $l \gg w>d^{14.15 .17}$, which permits to consider only transverse displacements of the beam. A longitudinal mechanical force controls the nonlinearity of the potential energy in the transverse direction. At low temperatures, the higher modes are frozen out and the fundamental mode can be treated independently. The resulting effective potential energy of the fundamental mode contains terms which are quadratic and quartic in the transverse amplitude $x$ of the fundamental mode. The externally applied $a c$-driving can be included on the same footing. The driving strength can be tuned within the fundamental mode description to the regime where non-linear effects come into play but the higher modes are still negligible (see below). One ends up with an effective single particle quantum mechanical time-dependent Hamiltonian for the fundamental mode amplitude $x$ acting as a position operator, i.e.,$^{14.15}$

$$
H_{S}(t)=-\frac{\hbar^{2}}{2 m^{*}} \frac{\partial^{2}}{\partial x^{2}}+\frac{\tilde{\alpha}}{2} x^{2}+\frac{\tilde{\beta}}{4} x^{4}+x f \cos (\omega t) .
$$

Here, $m^{*}$ is the effective mass of the beam. The parameters in the potential are obtained as ${ }^{14.15} \tilde{\alpha}=m^{*} \omega_{0}^{2} \equiv$ $m^{*} \bar{\omega}_{0}^{2}\left(\frac{\varepsilon_{c}-\varepsilon}{\varepsilon_{c}}\right)$ and $\tilde{\beta}=\frac{3 m^{*} \bar{\omega}_{0}^{2}}{d^{2}}$, where the frequency of the fundamental mode is given by $\bar{\omega}_{0}=\pi^{2} d \sqrt{\mathcal{Q} / \rho} /\left(l^{2} \sqrt{12}\right)$. $\mathcal{Q}$ is Young's elasticity modulus and $\rho$ the mass density of 
the material. The longitudinal force generates the strain $\varepsilon=\left(l-l_{0}\right) / l_{0}$ where $l_{0}$ is the equilibrium length of the beam. At the critical value $\varepsilon_{c}=-\pi^{2} d^{2} /\left(12 l^{2}\right)$ (for a rectangular beam), the system reaches a bifurcation point which is the well-known Euler instability. The effective potential for the fundamental mode then is purely quartic. Close to the Euler instability, both quadratic and quartic terms appear. We consider the case of a monostable potential, i.e., $\alpha>0$, which implies that the strain remains below its critical values, i.e., $\varepsilon<\varepsilon_{c}$. This situation is easier to be realized experimentally compared to the bistable potential $\alpha<0$. The ac-driving occurs with amplitude $f$ and frequency $\omega$. An upper limit $f_{\max }$ for the regime of validity of the fundamental mode description is given by the first harmonic threshold, i.e., $x_{0} f_{\max }<\hbar \omega_{1} \approx 3 \hbar \omega_{0}{ }^{14}$. We scale the Hamiltonian (11) with respect to the units in space and time, i.e., $x_{0}=\sqrt{\hbar /\left(m^{*} \omega_{0}\right)}$ and $t_{0}=1 / \omega_{0}$, respectively, yielding the energy scale $\hbar \omega_{0}$ and the dimensionless nonlinearity parameter $\beta \equiv \frac{3}{4\left(d / x_{0}\right)^{2}} \frac{\varepsilon_{c}}{\varepsilon_{c}-\varepsilon}$.

We include the effect of the environment phenomenologically by a set of harmonic oscillators which are bilinearly coupled to the system with the coupling constants $c_{j} \underline{18}$. The Hamiltonian is

$$
H_{B}=\frac{1}{2} \sum_{j} p_{j}^{2} / m_{j}+m_{j} \omega_{j}^{2}\left(x_{j}-\frac{c_{j}}{m_{j} \omega_{j}^{2}} x\right)^{2} .
$$

The bath is characterized by the spectral density

$$
I(\omega)=\frac{\pi}{2} \sum_{j} \frac{c_{j}^{2}}{m_{j} \omega_{j}} \delta\left(\omega-\omega_{j}\right)=m^{*} \gamma \omega e^{-\omega / \omega_{c}},
$$

where we have chosen the standard Ohmic form with damping constant $\gamma$ and with cut-off frequency $\omega_{c}$. The total Hamiltonian is $H(t)=H_{S}(t)+H_{B}$.

We focus on the case when the coupling to the bath is weak and use a standard Born-Markovian master equation ${ }^{19.20}$ which reads

$$
\dot{\rho}=-\frac{i}{\hbar}\left[H_{S}(t), \rho\right]+\mathcal{L}_{\text {rel }}(\rho)+\mathcal{L}_{\text {noise }}(\rho) .
$$

The commutator describes the coherent dynamics while the bath acts via the two superoperators for relaxation and noise, respectively,

$$
\begin{aligned}
\mathcal{L}_{\text {rel }}(\rho) & =-\frac{i \gamma}{2 \hbar}\left[x,[p, \rho]_{+}\right], \\
\mathcal{L}_{\text {noise }}(\rho) & =-\frac{1}{\hbar}[x,[Q, \rho]] .
\end{aligned}
$$

The operator

$$
Q=\int_{0}^{\infty} d \tau K(\tau) x_{\mathrm{H}}(t-\tau, t)
$$

involves the integral kernel

$$
K(\tau)=\frac{1}{\pi} \int_{0}^{\infty} d \omega I(\omega) \operatorname{coth}\left(\frac{\hbar \omega}{2 k_{B} T}\right) \cos \omega t
$$

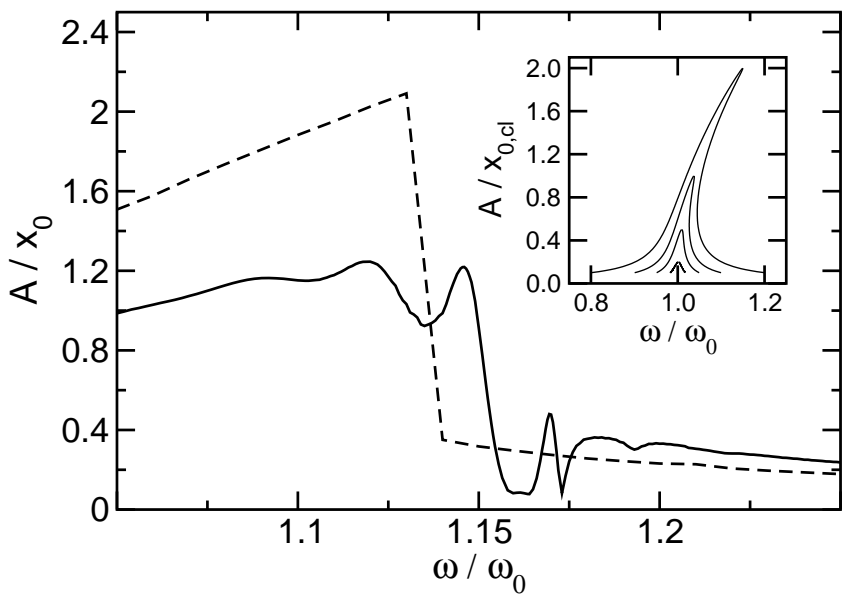

FIG. 1: Amplitude $A$ of the expectation value $\langle x(t)\rangle$ for varying the driving frequency $\omega$. Parameters are $k_{B} T=$ $0.1 \hbar \omega_{0}, \beta=0.1, f=0.1 \hbar \omega_{0} / x_{0}, \gamma=0.005 \omega_{0}$. Dashed line: Results of the classical Duffing oscillator at $T=0$ with the remaining parameters being the same. Inset: Amplitude $A$ of the classical Duffing oscillator for varying driving frequencies. The driving strength $f$ increases from bottom to top.

with $T$ being temperature and the position operator $x_{\mathrm{H}}(t-\tau, t)$ in the Heisenberg picture. This master equation can be solved numerically to obtain $\rho(t)$ and the expectation value

$$
\langle x(t)\rangle=\operatorname{tr}(\rho(t) x) .
$$

In the stationary limit, $\langle x(t)\rangle$ shows oscillations with the frequency being the external driving frequency $\omega$ (plus a phase shift) and with amplitude $A$. This amplitude is the quantity of interest that allows to compare to the classical counterpart.

\section{NON-LINEAR RESPONSE OF THE NANORESONATOR}

With the oscillation amplitude $A$ at hand, we can investigate the non-linear response of the nanoresonator on varying driving frequencies $\omega$. The classical analogue shows a well-known rich dynamics including chaos and driving induced bistability. Without noise, we obtain the Duffing oscillator ${ }^{17,21}$. Its response on varying the driving frequency can be calculated perturbatively ${ }^{17}$ and is shown in Fig. 10 (dashed line and inset). For weak driving, the standard Lorentzian resonance of a harmonic oscillator occurs. For stronger driving, the system enters the nonlinear regime, the resonance curve bends over and an effective bistability is induced yielding a hysteretical response. If Gaussian white noise is added, the range of bistability and therefore hysteresis is reduced 22 since the system escapes more easily from the metastable state by thermal hopping. 


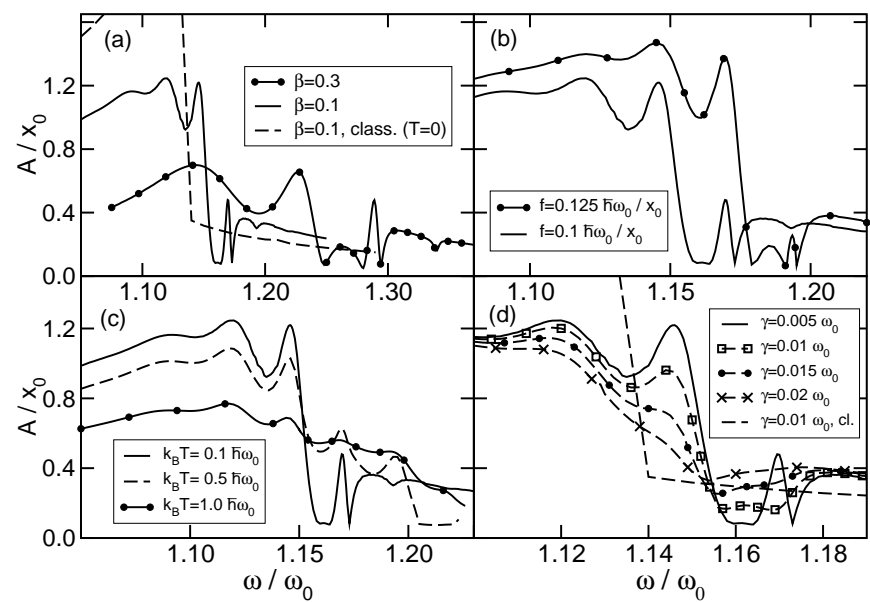

FIG. 2: Amplitude $A$ for varying the driving frequency $\omega$ and for different choices of (a) the nonlinearity coefficient $\beta$, (b) driving intensity $f$, (c) temperature $T$ and (d) damping strength $\gamma$. The other parameters are kept fixed according to $\beta=0.1, f=0.1 \hbar \omega_{0} / x_{0}, k_{B} T=0.1 \hbar \omega_{0}$ and $\gamma=0.005 \omega_{0}$, respectively. Dashed line in (a) and (d): same as in Fig. 1]

For the quantum case, one can ask whether a signature of the induced bistability still appears in the response. For a truly bistable potential, tunneling leads to a reduction of hysteresis 23 . Also, resonant tunneling through the potential barrier leads to characteristic steps in the hysteresis cycle 24 . However, the potential in the Hamiltonian Eq. (1) is monostable.

We have calculated the amplitude $A$ of the steady-state oscillations for varying driving frequencies $\omega$. The result is shown in Fig. 1] The characteristic profile consists of a series of peaks and dips. The resonances are sharper for higher frequencies. For lower frequencies, the broad peaks overlap strongly and lead to a shoulder-like profile which is similar to the classical result (dashed line in Fig. 11). The locations of the resonances are dominated by the parameters of the undamped driven system, see Fig. 2a and $2 \mathrm{~b}$. On the other hand, by changing the parameters characterizing the bath (temperature $T$ and damping strength $\gamma$ ) the shape of the resonances is modified. As can be seen in Fig. 2r: and 21, for increasing temperature and damping, the sharp resonances are smeared out and finally fade out when the quantum coherence is suppressed. This indicates that the resonances are related to resonant multi-phonon excitations in the driven nonlinear system.

\section{RESONANT MULTI-PHONON EXCITATION}

In order to show this, we exploit the periodicity in time of $H_{S}(t)$ and calculate the quasienergy (Floquet) spectrum for varying driving frequencies $\omega^{25,26}$. To this

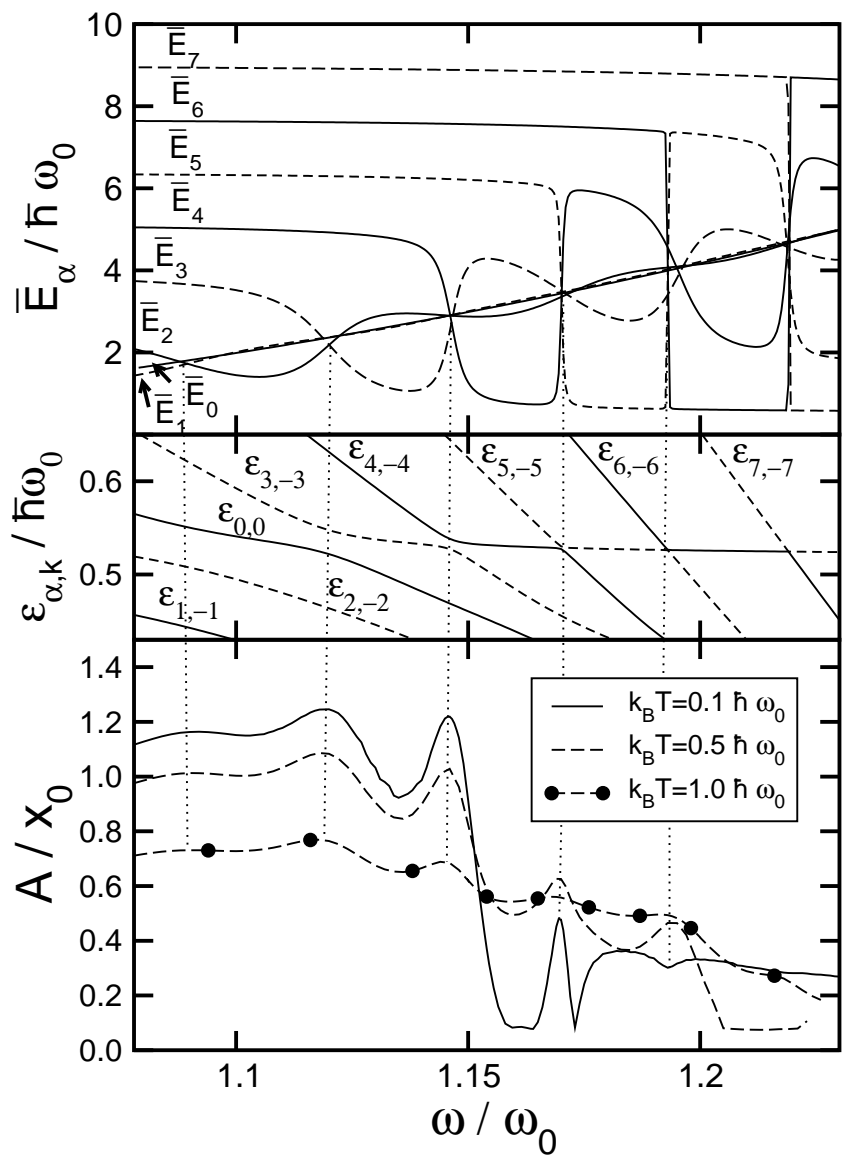

FIG. 3: Average energies $\bar{E}_{i}$ (top), quasienergy levels $\varepsilon_{\alpha, k}$ (middle) and amplitude $A$ of the fundamental mode for varying driving frequencies $\omega$. The remaining parameters are $\beta=0.1, f=0.1 \hbar \omega_{0} / x_{0}$ and $\gamma=0.005 \omega_{0}$.

end, we solve the equation

$$
\left[H_{S}(t)-i \hbar \frac{\partial}{\partial t}\right]\left|\phi_{\alpha}(t)\right\rangle=\varepsilon_{\alpha}\left|\phi_{\alpha}(t)\right\rangle,
$$

where the quasienergies $\varepsilon_{\alpha}$ are defined up to a multiple integer of $\hbar \omega$. This means that the state $\left|\phi_{\alpha}^{(n)}(t)\right\rangle=$ $e^{i n \omega t}\left|\phi_{\alpha}(t)\right\rangle$ is also an eigenstate of the Floquet Hamiltonian, but with the eigenvalue

$$
\varepsilon_{\alpha, n}=\varepsilon_{\alpha}+n \hbar \omega .
$$

Since the quasienergies do not allow for global ordering, we have also calculated the mean energies averaged over one driving period

$$
\bar{E}_{\alpha}=\sum_{n}\left(\varepsilon_{\alpha}+n \hbar \omega\right)\left\langle c_{\alpha, n} \mid c_{\alpha, n}\right\rangle,
$$

where the $\left|c_{\alpha, n}\right\rangle$ are the Fourier components of the Floquet states 25 , which can be obtained as

$$
\left|c_{\alpha, n}\right\rangle=\frac{\omega}{2 \pi} \int_{0}^{2 \pi / \omega} d t e^{i n \omega t}\left|\phi_{\alpha}(t)\right\rangle .
$$




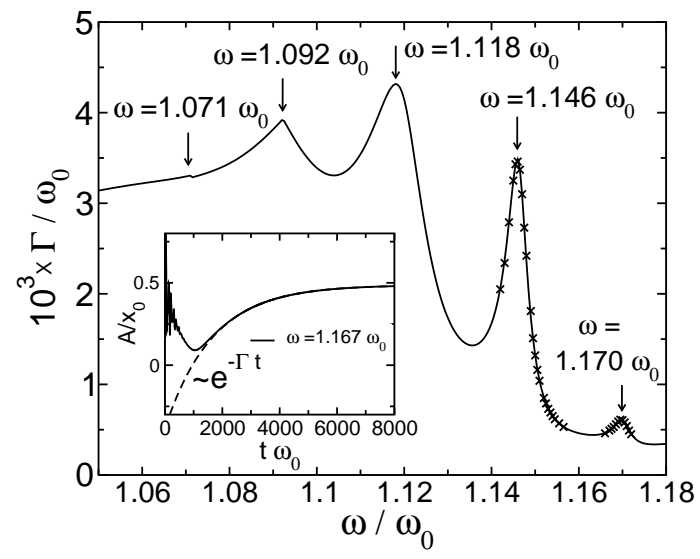

FIG. 4: Decay rate $\Gamma$ for the slow dynamics of the amplitude $A$ for approaching the steady state. Symbols: Solution from the numerical iteration of the Born Markovian master equation, solid line: Smallest non-zero eigenvalue of the rate matrix of an improved Floquet Markovian master equation (see text). Inset: time-resolved dynamics of $A$ for $\omega=1.167 \omega_{0}$ (solid line). The dashed line shows a fit to an exponential $e^{-\Gamma t}$. Here, $\beta=0.1, f=0.1 \hbar \omega_{0} / x_{0}, k_{B} T=0.1 \hbar \omega_{0}$ and $\gamma=0.005 \omega_{0}$.

For the harmonic case $(\beta=0)$, the quasienergies are all degenerate for infinitesimal driving. The strong driving and the nonlinear potential lift this degeneracy. As follows from the result shown in Fig. 3 the quasienergies show avoided level crossings (and the mean energies show exact crossings) for particular values of the driving frequency $\omega$. To these (anti-)crossings correspond the particular resonances in the frequency-dependence of the amplitude $A$. The resonances occur when the (undamped) quantum system absorbs an integer multiple of $\hbar \omega$. The shape of the resonances is related to the splitting of the quasienergy levels at the avoided level crossings. For lower frequencies, the level splitting is quite large. Moreover, the avoided crossings are not well separated in this regime. This implies that the resonances are broad and strongly overlap yielding the shoulder-like behavior of the response profile. For larger $\omega$, the avoided level crossings become well separated and the energy splittings decrease. Note that the resonances occur despite the fact that the thermal energy is larger than the quasienergy level splittings at the avoided level crossing. In turn, this leads to sharp resonances in the amplitude. The physics of avoided quasi-energy level crossings has been discussed in detail in Ref. 27.

\section{DYNAMICALLY INDUCED BISTABILITY AND RESONANT TUNNELING}

The bistability of the steady state of the classical Duffing oscillator does not survive in the quantum system since it escapes the metastable state asymptotically via tunneling 24 . Nevertheless, we find signatures of bistabil- ity and tunneling if we consider how the steady state is reached. For this, we show in the inset of Fig. 4 the amplitude $A$ (local maxima of the vibrations) for increasing time (starting with the ground state of the undriven oscillator as the initial state). We observe fast oscillations at short times. They decay on a time scale $\gamma^{-1}$ which reflects intrawell relaxation in the metastable state. Then, starting from a metastable state at intermediate times, a slow exponential decay towards the asymptotically stable state can be observed. By fitting to an exponential, we extract the decay rate $\Gamma$ for various driving frequen-

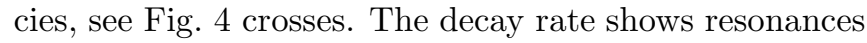
at the same values of the frequencies where the avoided crossings of the quasienergy levels occur (see arrows). This slow dynamics can be identified as quantum tunneling in a dynamically induced bistable effective potential (see also Ref. 28). The peaks in $\Gamma$ indicate resonant tunneling ${ }^{24}$ from the meta- to the globally stable state both of which are dynamically induced.

The rate for tunneling out of the metastable well can be obtained directly from an improved Floquet-Markovian master equation ${ }^{20}$. In the moderate rotating wave approximation, the time-independent master equation

$$
\dot{\rho}_{\alpha \beta}(t)=-\frac{i}{\hbar}\left(\varepsilon_{\alpha}-\varepsilon_{\beta}\right) \rho_{\alpha \beta}(t)+\sum_{\alpha^{\prime} \beta^{\prime}} \mathcal{L}_{\alpha \beta, \alpha^{\prime} \beta^{\prime}} \rho_{\alpha^{\prime} \beta^{\prime}}(t)
$$

in the Floquet basis can be established. The transition rates containing the influence of the dissipative bath $\operatorname{read}^{20}$

$$
\begin{aligned}
\mathcal{L}_{\alpha \beta, \alpha^{\prime} \beta^{\prime}}= & \sum_{n}\left(N_{\alpha \alpha^{\prime}, n}+N_{\beta \beta^{\prime}, n}\right) X_{\alpha \alpha^{\prime}, n} X_{\beta^{\prime} \beta,-n} \\
& -\delta_{\beta \beta^{\prime}} \sum_{\beta^{\prime \prime}, n} N_{\beta^{\prime \prime} \alpha^{\prime}, n} X_{\alpha \beta^{\prime \prime},-n} X_{\beta^{\prime \prime} \alpha^{\prime}, n} \\
& -\delta_{\alpha \alpha^{\prime}} \sum_{\alpha^{\prime \prime}, n} N_{\alpha^{\prime \prime} \beta^{\prime}, n} X_{\beta^{\prime} \alpha^{\prime \prime},-n} X_{\alpha^{\prime \prime} \beta, n} \cdot(14)
\end{aligned}
$$

Here, the coefficients are given by

$$
\begin{aligned}
& N_{\alpha \beta, n}=N\left(\varepsilon_{\alpha}-\varepsilon_{\beta}+n \hbar \omega\right), N(\varepsilon)=\frac{m^{*} \gamma \varepsilon}{\hbar^{2}} \frac{1}{e^{\varepsilon / k_{B} T}-1} \\
& X_{\alpha \beta, n}=\frac{\omega}{2 \pi} \int_{0}^{2 \pi / \omega} d t e^{-i n \omega t}\left\langle\phi_{\alpha}(t)|x| \phi_{\beta}(t)\right\rangle .
\end{aligned}
$$

The time-independent rate coefficients (14) can be written as a matrix which can readily be diagonalized numerically. We find a clear separation of time-scales where the smallest non-zero eigenvalue indicates a slow tunneling dynamics by which the stationary state is approached. The solid line of Fig. [4 shows the smallest non-zero eigenvalue of the corresponding rate matrix which was obtained by direct numerical diagonalization using a basis of 8 Floquet eigenstates. The result agrees well with that from the numerical iteration of the Born-Markovian master equation. Note again the analogy to resonant tunneling in a static double-well potential ${ }^{24}$. The role of the eigenenergies in the static case is now played by the 
quasienergies $\varepsilon_{\alpha}$ determining the coherent dynamics, see Eq. (13). In both cases, the avoided (quasi-)energy level crossings are the origin of resonant tunneling from the metastable towards the globally stable state. Nevertheless, the incoherent part of Eq. (13) is crucial to observe the resonant tunneling in this driving induced bistability.

\section{CONCLUSIONS}

In conclusion, we have found resonances in the amplitude of the fundamental mode of a nanomechanical resonator which is driven into its non-linear regime. They occur for particular values of the driving frequency and are explained in terms of quantum mechanical resonant multi-phonon excitations between quasienergy states. This response profile is generic for the quantum system and is absent in the analogous classical system. In addition, we have identified resonant tunneling in a dynamically induced bistable effective potential. Since oscillation amplitudes of nanomechanical resonators can be detected with currently available experimental techniques, this macroscopic quantum effect should be measurable in the near future. A promising approach seems to be to couple a superconducting single-electron transistor capacitively to the resonator ${ }^{11}$ allowing an ultra-high sensitivity. Indeed, a fundamental frequency of $19 \mathrm{MHz}$ has been reported ${ }^{11}$ implying that quantum effects occur below a temperature of a few $\mathrm{mK}$.
Most interestingly, the reported quality factors around $Q=40000$ seem to be very promising since we predict quantum effects at even smaller $Q=\omega_{0} / \gamma$, see above.

Finally, we note that our model of a driven anharmonic mono-stable quantum oscillator is a generic model which finds applications in various other fields of physics. One important application is related to the non-destructive readout for superconducting flux or charge qubits 29 . There, a dc-SQUID which is inductively coupled to the qubit is driven by an ac bias current such that the SQUID remains in its superconducting state (for this, the amplitude of the ac current has to be smaller than the critical current of the SQUID). The Josephson inductance of the SQUID carrying information on the qubit state is measured for varying the frequency of the ac-current. In terms of our model, the SQUID provides a sinusoidal potential for the superconducting phase acting as a single quantum mechanical particle. The particle is initially localized in one potential minimum. The ac bias current provides the external driving and for stronger driving, the particle experiences the nonlinearity of the potential well. The distinct resonances in the nonlinear response which we have found might help to increase the efficiency of the read-out process since they are sharper than the common linear resonance.

\section{Acknowledgments}

We thank Peter Hänggi for helpful discussions. We acknowledge support by the DFG-SFB/TR 12 .
1 M. L. Roukes, Physics World 14, 25 (2001); H. G. Craighead, Science 290, 1532 (2000); A. N. Cleland, Foundations of Nanomechanics, (Berlin, Springer, 2003).

2 A. N. Cleland and M. L. Roukes, Appl. Phys. Lett. 69, 2653 (1996); A. N. Cleland and M. L. Roukes, Nature 392, 161 (1998).

3 H. Krömmer et al., Europhys. Lett. 50, 101 (2000).

4 A. Erbe et al., Appl. Phys. Lett. 77, 3102 (2000).

${ }^{5}$ F. W. Beil et al., Nanotechnology 14, 799 (2003).

6 A. N. Cleland, M. Pophristic and I. Ferguson, App. Phys. Lett. 79, 2070 (2001).

7 C. T.-C. Nguyen, A.-C. Wong and H. Ding, Dig. Tech. Pap.-IEEE Int. Solid-State Circuits Conf. 448, 78 (1999).

8 X. M. H. Huang, C. A. Zorman, M. Mehregany and M. Roukes, Nature 421, 496 (2003).

${ }^{9}$ R. G. Knobel and A. N. Cleland, Nature 424, 291 (2003).

10 A. Husain et al., Appl. Phys. Lett. 83, 1240 (2003).

11 M. D. LaHaye, O. Buu, B. Camarota, and K. Schwab, Science 304, 74 (2004).

12 B. Babic et al., Nano Lett. 3, 1577 (2003).

13 C. Li and T.-W. Chou, App. Phys. Lett. 84, 121 (2004).

14 S. M. Carr, W. E. Lawrence and M. N. Wybourne, Phys. Rev. B 64, 220101 (2001); Physica B 316-317, 464 (2002).

15 P. Werner and W. Zwerger, Europhys. Lett. 65, 158 (2004).

16 P. Poncharal, Z. L. Wang, D. Ugarte, and W. de Heer, Science 283, 1513 (1999).

17 A. H. Nayfeh and D. T. Mook, Nonlinear Oscillations (Wiley, New York, 1979).
18 U. Weiss, Quantum Dissipative Systems (World Scientific, Singapore, 2nd ed., 1999).

19 W. H. Louisell, Quantum Statistical Properties of Radiation (Wiley, New York, 1973).

20 S. Kohler, T. Dittrich, and P. Hänggi, Phys. Rev. E 55, 300, (1997).

21 J. Guckenheimer and Ph. Holmes, Nonlinear oscillations, dynamical systems, and bifurcations of vector fields (Springer, New York, 1983).

22 S. Datta and J. K. Bhattacharjee, Phys. Lett. A 283, 323 (2001).

23 M. Thorwart and P. Jung, Phys. Rev. Lett. 78, 2503 (1997).

24 M. Thorwart, P. Reimann, P. Jung and R. F. Fox, Phys. Lett. A 239, 233 (1998); Chem. Phys. 235, 61 (1998).

25 M. Grifoni and P. Hänggi, Phys. Rep. 304, 229 (1998).

26 A. G. Fainshtein, N. L. Manakov, and L. P. Rapoport, J. Phys. B 11, 2561 (1978).

27 S. Kohler, R. Utermann, P. Hänggi, and T. Dittrich, Phys. Rev. E 58, 7219 (1998).

28 M. I. Dykman and V. N. Smelyanskii, Sov. Phys. JETP 67, 1769 (1988).

29 A. Lupascu et al., Phys. Rev. Lett. 93, 177006 (2004). 